\title{
Changing trends in the bacteriological profiles and antibiotic susceptibility in neonatal sepsis at a tertiary children's hospital of China
}

\author{
Xiao-Juan Tang, Bin Sun, Xin Ding, Hong Li, Xing Feng \\ Neonatal Intensive Care Unit, Department of Neonatology, Children's Hospital of Soochow University, Suzhou, China \\ Contributions: (I) Conception and design: X Feng, XJ Tang; (II) Administrative support: B Sun, X Ding; (III) Provision of study materials or patients: \\ XJ Tang, H Li; (IV) Collection and assembly of data: XJ Tang, H Li; (V) Data analysis and interpretation: XJ Tang; (VI) Manuscript writing: All \\ authors; (VII) Final approval of manuscript: All authors. \\ Correspondence to: Professor Xing Feng, PhD. Chairman of the Pediatrics Branch of Jiangsu Medical Association, Department of Neonatology, \\ Children’s Hospital of Soochow University, Suzhou, China. Email: fengxing@suda.edu.cn.
}

\begin{abstract}
Background: Sepsis is a major cause of neonatal morbidity and mortality in developing countries, and early-onset sepsis has poor outcomes. The causative bacteria vary depending on the geographical location of the hospital. This study aimed to determine the changing trends of causative bacteria and antibiotic susceptibility in the past decade.

Methods: This study retrospectively analyzed the blood culture of positive cases of early-onset sepsis admitted to the neonatal intensive care unit of our hospital between 2009 and 2018. The cases were divided into two phases, i.e., phase I (2009 to 2013) and phase II (2014 to 2018). Changing trends in the bacteriological profiles and antibiotic susceptibility were recorded and analyzed.

Results: A total of 1,479 causative bacteria were detected. Gram-positive bacteria were isolated in $74.92 \%$ of the cases, and coagulase-negative Staphylococci (CoNS) (63.22\%) was identified as the common isolate. Klebsiella pneumoniae (10.01\%) followed by Escherichia coli (8.72\%) were the dominant Gram-negative bacteria. Comparative analysis showed a significant reduction in CoNS. Among Gram-negative bacteria, $K$. pneumoniae was initially predominant but was replaced by E. coli in phase II. Gram-positive bacteria showed relatively high susceptibility to aminoglycosides and quinolones. $K$. pneumoniae exhibited higher resistance to cephalosporin compared with E. coli. Reduced sensitivity against the first- and secondgeneration antibiotics was observed in phase II.

Conclusions: The etiological profile of neonatal sepsis (NS) has undergone a significant change in the last decade. Antibiotic resistance has increased, and continuous surveillance for antibiotic susceptibility is required to ensure efficient therapeutic outcomes.
\end{abstract}

Keywords: Bacteriocins; newborn; sepsis

Submitted Apr 06, 2020. Accepted for publication Oct 30, 2020.

doi: $10.21037 /$ tp-20-115

View this article at: http://dx.doi.org/10.21037/tp-20-115

\section{Introduction}

The microbial etiology of neonatal sepsis (NS) varies according to the geographical location of hospitals; as such, developing therapeutic interventions is challenging. NS is defined as a systemic infection, usually bacterial, viral, or fungal, that may occur with/without signs and symptoms of infection during the first 4 weeks of life (1). Early-onset sepsis is defined as sepsis occurring less than 7 days of age and often has worse prognosis than late-onset sepsis ( $\geq 7$ days of age). Despite the improvements in NS treatment strategies, it remains a major threat to the health of neonates, particularly in developing countries (2). Several 
studies have found changing trend in the bacterial etiology of NS in different periods (3-5). Early identification and treatment of NS are challenging due to variations in the microbial etiology of regions where hospitals are located. Periodic epidemiological surveys of NS can identify commonly encountered pathogens and their antibiotic susceptibility patterns in a neonatal setting.

Antibiotics are the standard therapy for high-risk patients with NS, including preterm infants and those who have ruptured membranes and low body weight. The diversity of organisms causing sepsis changes over time, which poses a challenge for selection of antibiotics for empirical treatment (3-6). Additionally, prolonged antibiotic exposure leads to poor prognosis in neonates. This study focused on determining the causative bacteria and antibiotic susceptibility of early-onset sepsis due to the differences in the etiology between early-onset and late-onset sepsis. Here, we investigated the trend in the etiology of NS and their antibiotic susceptibility pattern over the last decade in our hospital. We present the following article in accordance with the STROBE reporting checklist (available at http:// dx. doi. org/10. 21037/tp-20-115).

\section{Methods}

\section{Pretreatment evaluation}

We obtained data on blood cultures of patients with earlyonset sepsis ( $<7$ days of age) who were admitted to the neonatal intensive care unit at Children's Hospital of Soochow University between Jan 2009 and Dec 2018. The cases were divided into two phases, i.e., phase I (2009 to 2013) and phase II (2014 to 2018). Bacterial and demographic data were obtained and compared with microbial databases. The study was conducted in accordance with the Declaration of Helsinki (as revised in 2013). The study was approved by the Clinical Trial Ethics Review Committee of Children's Hospital of Soochow University (No. 2019LW019) and individual consent for this retrospective analysis was waived.

We collected demographic and microbial data from ward admission and laboratory registers and validated these against the electronic microbial database. Blood cultures of patients who were potentially septic were assessed. The clinical outcome and duration of hospital stay were not assessed. Bacterial colonization data from vaginal swabs and antibiotic therapy during neonate delivery were unavailable.
NS was defined as neonates presenting with at least one of the following symptoms: fever (rectal temperature $\geq 38{ }^{\circ} \mathrm{C}$ ) or hypothermia (rectal temperature $\leq 36{ }^{\circ} \mathrm{C}$ ), white blood cell counts $\geq 30,000 / \mathrm{mm}$ or $<5,000 / \mathrm{mm}$ or $>25 \%$ immature cells, convulsions, lethargy, feeding or breathing problem, hypoglycemia, bulging fontanels, vomiting, and jaundice.

\section{Blood cultures}

Blood was sampled from the peripheral vein under aseptic conditions before antibiotic interventions. Blood $(2 \mathrm{~mL})$ was inoculated into the brain-heart infusion broth at $37^{\circ} \mathrm{C}$. Sub-cultures were produced on blood and MacConkey's agar following 24 and 48 h of growth. Bacterial growth was identified through colony counting, Gram staining, slide agglutination, and biochemical properties. If no growth was evident after $48 \mathrm{~h}$, then the growth period was extended to 7 days before being confirmed as sterile. Mixed bacterial flora or diphtheroid growth represented contamination. Coagulase-negative Staphylococci (CoNS) was considered pathogen only when isolated in paired cultures. All isolates were analyzed. Antimicrobial susceptibility was assessed using KirbyBauer disk diffusion method. Data were interpreted based on the criteria of the Clinical and Laboratory Standard Institute. The cultures with aerobic spores were considered contaminated. Gram-positive bacteria were tested against the following antimicrobials: oxacillin, erythromycin, ciprofloxacin, rifampin, linezolid, moxifloxacin, penicillin G, gentamicin, tetracycline, tigecycline, cefoxitin, vancomycin, and levofloxacin. Gram-negative bacteria were tested against the following antimicrobials: amikacin, ampicillin, aztreonam, ertapenem, ciprofloxacin, meropenem, piperacillin, piperacillin/tazobactam, gentamicin, cefepime, cefuroxime, cefoperazone/sulbactam, ceftriaxone, cefotaxime, ceftazidime, cefotetan, cefoxitin, imipenem, and levofloxacin.

\section{Data analysis}

The results of the laboratory investigations were recorded in a proforma and analyzed using SPSS version 17.0. Descriptive statistics were used to describe data distribution. Chi-square test was performed for categorical variables. $\mathrm{P}<0.01$ was considered statistically significant. 
Table 1 Changing trends in distributions of bacterial isolates between the two phases

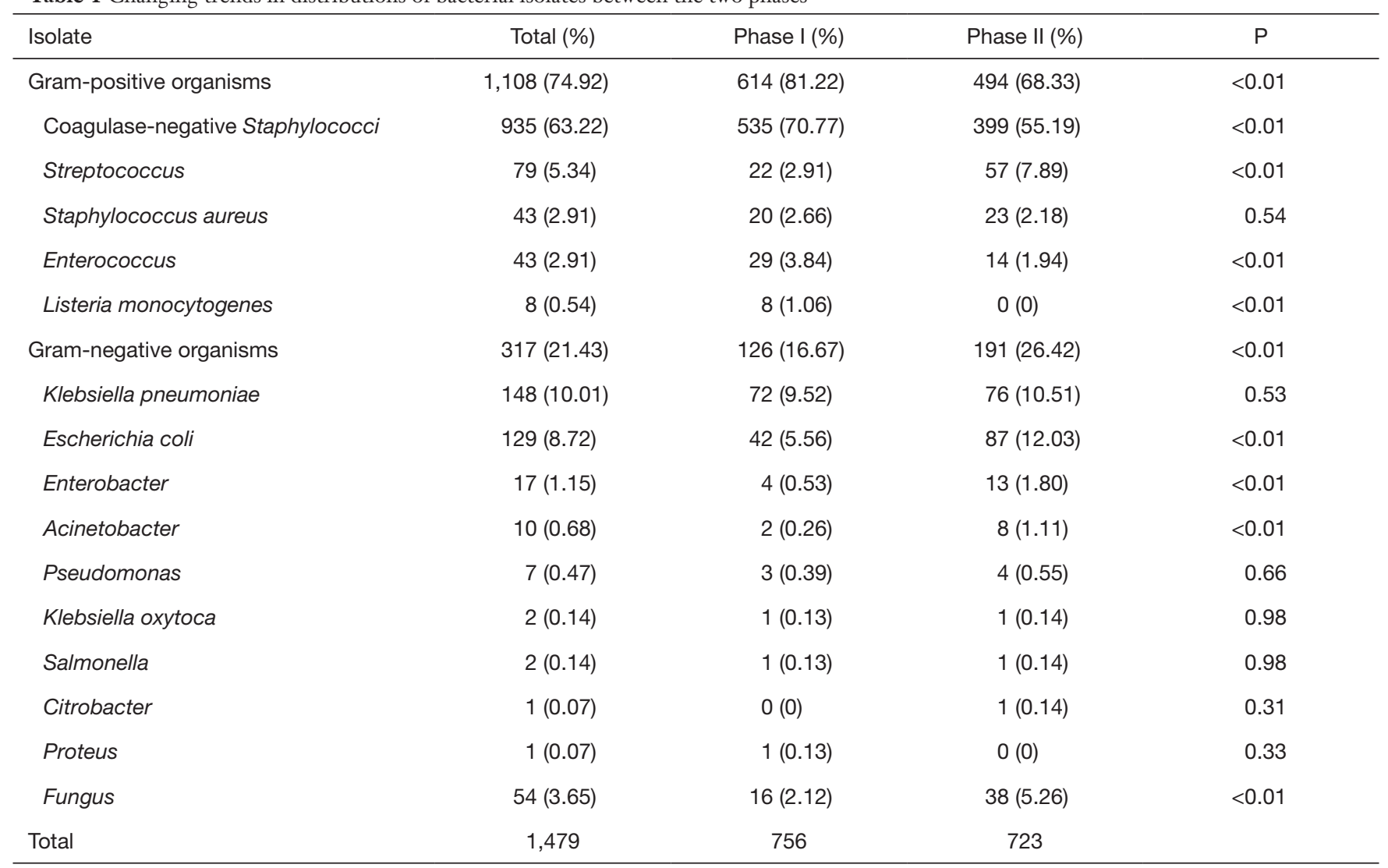

\section{Results}

\section{Isolated organisms}

Table 1 provides the detailed etiology of the 1,479 isolates, including Gram-positive bacteria (74.92\%), Gram-negative bacteria $(21.43 \%)$, and fungi $(3.65 \%)$. CoNS were the most common organisms accounting for $63.15 \%$ of the isolates and mainly comprised S. epidermidis $(57.49 \%$, i.e., 537/934), S. hemolyticus (20.56\%, i.e., 192/934), and S. hominis (10.49\%, i.e., 98/934). Common Gram-negative bacteria were Klebsiella pneumoniae (10.01\%) and Escherichia coli $(8.72 \%)$. Among other Gram-positive bacteria, Streptococcus accounted for $5.34 \%$, Enterococcus accounted for $2.91 \%$, and $S$. aureus accounted for $2.91 \%$. The table also shows the comparative analysis of the changing trends in bacteriological profiles in the two phases. Gram-positive bacteria were still the predominant isolates. However, the proportion decreased in phase II $(\mathrm{P}<0.01)$. CoNS were the most common organisms among Gram-positive bacteria, but their proportion significantly decreased in phase II $(\mathrm{P}<0.01)$. K. pneumoniae was the predominant pathogen among Gram-negative bacteria in phase I. However, E. coli had replaced K. pneumoniae as the prevalent Gram-negative bacteria in phase II.

\section{Antibiotic susceptibility}

Table 2 shows the analysis of the antibiotic susceptibility of CoNS and S. aureus. CoNS were more susceptible to commonly used non- $\beta$-lactam antibiotics compared with oxacillin and penicillin. CoNS showed $95.1 \%$ resistance to penicillin $\mathrm{G}$ and $78.1 \%$ resistance to oxacillin. The percentages of susceptibility were $100 \%$ against linezolid, tigecycline, and teicoplanin; $76.96 \%$ against cefoxitin; and $58.04 \%$ against levofloxacin. S. aureus was more susceptible to commonly used non- $\beta$-lactam antibiotics compared with penicillin and cefoxitin. The results showed that S. aureus was fully susceptible to moxifloxacin, tigecycline, teicoplanin, and vancomycin followed by gentamicin (96.97\%) and ciprofloxacin (91.30\%), but less sensitive to cefoxitin $(28.57 \%)$ and penicillin $(18.18 \%)$. Table 3 shows the susceptibility assessment of K. pneumonia and 
Table 2 Antibiotic susceptibility of the major gram-positive bacteria

\begin{tabular}{lcc}
\hline Antimicrobial & CoNS (\%) & S. aureus (\%) \\
\hline Oxacillin & $143 / 653(21.90)$ & $26 / 33(78.79)$ \\
Cotrimoxazole & $394 / 653(60.34)$ & $31 / 33(93.94)$ \\
Erythromycin & $152 / 653(23.23)$ & $18 / 33(54.55)$ \\
Ciprofloxacin & $265 / 453(58.80)$ & $21 / 23(91.30)$ \\
Rifampicin & $626 / 653(95.87)$ & $33 / 33(100.00)$ \\
Linezolid & $652 / 652(100.00)$ & $33 / 33(100.00)$ \\
Moxifloxacin & $502 / 653(76.88)$ & $33 / 33(100.00)$ \\
Penicillin G & $32 / 653(4.90)$ & $6 / 33(18.18)$ \\
Gentamicin & $518 / 653(79.33)$ & $32 / 33(96.97)$ \\
Tetracycline & $498 / 653(76.26)$ & $28 / 33(84.85)$ \\
Tigecycline & $378 / 378(100.00)$ & $21 / 21(100.00)$ \\
Teicoplanin & $276 / 276(100.00)$ & $11 / 11(100.00)$ \\
Cefoxitin & $157 / 204(76.96)$ & $6 / 21(28.57)$ \\
Vancomycin & $651 / 652(99.85)$ & $33 / 33(100.00)$ \\
Levofloxacin & $379 / 653(58.04)$ & $30 / 33(90.91)$ \\
\hline CoNS, coagulase-negati) & \\
\hline
\end{tabular}

CoNS, coagulase-negative Staphylococci.

E. coli. For $\beta$-lactam antibiotics, K. pneumoniae showed the maximum susceptibility to ertapenem (100\%), piperacillin/ tazobactam (86.61\%), meropenem (85.71\%), and imipenem $(82.93 \%)$ and had high resistance to ampicillin (100\%), piperacillin (93.75\%), cefuroxime (85.71\%), and cefotaxime $(85.0 \%)$. For non- $\beta$-lactam antibiotics, K. pneumoniae showed the maximum susceptibility to aminoglycosides and quinolones including amikacin (100\%), ciprofloxacin (96.0\%), and levofloxacin (93.90\%). E. coli demonstrated high susceptibility to aminoglycosides, carbapenem, and quinolones but low susceptibility to ampicillin (27.43\%) and piperacillin (28.57\%). E. coli maintained relatively high susceptibility to most third-generation cephalosporins.

Table 4 presents the comparison of the antibiotic susceptibility of CoNS isolated between 2009 and 2018. The susceptibility of the isolates to ciprofloxacin, tetracycline, and levofloxacin significantly increased in phase II compared with that in phase $\mathrm{I}(\mathrm{P}<0.01)$. By contrast, the antibiotic susceptibility of CoNS against cefoxitin decreased from $81.08 \%$ in phase I to $72.04 \%$ in phase II $(\mathrm{P}<0.01)$. Table 5 summarizes the comparison of the antibiotic susceptibility of $S$. aureus. No significant difference in the susceptibility of the isolates was observed during the two phases. K. pneumoniae showed similar
Table 3 Antibiotic susceptibility of the major gram-negative bacteria

\begin{tabular}{|c|c|c|}
\hline Antimicrobial & K. pneumoniae (\%) & E. coli $(\%)$ \\
\hline Amikacin & 98/98 (100.00) & $116 / 116(100.00)$ \\
\hline Ampicillin & $0 / 123(0)$ & $31 / 113(27.43)$ \\
\hline Aztreonam & $42 / 100(42.0)$ & 76/92 (82.61) \\
\hline Ertapenem & $83 / 83(100.00)$ & 92/92 (100.00) \\
\hline Cotrimoxazole & $61 / 100(61.00)$ & 42/92 (45.65) \\
\hline Ciprofloxacin & $96 / 100(96.00)$ & $55 / 92(59.78)$ \\
\hline Meropenem & $36 / 42$ (85.71) & 64/65 (98.46) \\
\hline Piperacillin & $4 / 64(6.25)$ & $18 / 63(28.57)$ \\
\hline $\begin{array}{l}\text { Piperacillin/ } \\
\text { tazobactam }\end{array}$ & $110 / 127$ (86.61) & $114 / 117(97.44)$ \\
\hline Gentamicin & $113 / 123(91.87)$ & 67/113 (59.29) \\
\hline Cefepime & $45 / 120(37.50)$ & $94 / 113(83.19)$ \\
\hline Cefuroxime & $10 / 70(14.29)$ & $32 / 52(61.54)$ \\
\hline $\begin{array}{l}\text { Cefoperazone/ } \\
\text { sulbactam }\end{array}$ & 79/134 (58.96) & $101 / 112(90.18)$ \\
\hline Ceftriaxone & 20/99 (20.20) & $53 / 83(63.86)$ \\
\hline Cefotaxime & $6 / 40(15.00)$ & $22 / 39(56.41)$ \\
\hline Ceftazidime & $42 / 123(34.15)$ & 91/114 (79.82) \\
\hline Cefotetan & $84 / 100(84.00)$ & 88/91 (96.70) \\
\hline Cefoxitin & $96 / 123$ (78.05) & $105 / 110(95.45)$ \\
\hline Imipenem & 68/82 (82.93) & $108 / 111(97.30)$ \\
\hline Levofloxacin & 77/82 (93.90) & 80/109 (73.39) \\
\hline
\end{tabular}

resistance to ampicillin and piperacillin. The resistance against second-and third-generation cephalosporins was significantly higher in phase II than that in phase I. $K$. pneumoniae showed high sensitivity toward amikacin and gentamicin among aminoglycosides throughout the study. The resistance to carbapenems and $\beta$-lactamase inhibitors significantly increased in phase II (Table 6). Table 7 depicts the resistance patterns of $E$. coli over time. The resistance to ampicillin and piperacillin was comparable over the study period, whereas that to carbapenems and $\beta$-lactamase inhibitors increased gradually.

\section{Discussion}

Despite recent advances in health care, morbidity and 
Table 4 Trend of antibiotic susceptibility for CoNS between the two phases

\begin{tabular}{|c|c|c|c|}
\hline Antimicrobial & Phase I (\%) & Phase II (\%) & $P$ \\
\hline Cotrimoxazole & 239/399 (59.90) & $155 / 254(61.02)$ & 0.78 \\
\hline Erythromycin & 87/399 (21.80) & 65/254 (25.59) & 0.26 \\
\hline Ciprofloxacin & $105 / 199(52.76)$ & 160/254 (62.99) & $<0.01$ \\
\hline Linezolid & $398 / 398$ (100.00) & $254 / 254(100.00)$ & \\
\hline Moxifloxacin & $301 / 399$ (75.44) & $201 / 254(79.13)$ & 0.28 \\
\hline Penicillin G & 21/399 (5.26) & $11 / 254(4.33)$ & 0.59 \\
\hline Gentamicin & 312/399 (78.20) & 206/254 (81.10) & 0.37 \\
\hline Teicoplanin & $112 / 112(100.00)$ & $164 / 164(100.00)$ & \\
\hline Cefoxitin & $90 / 111$ (81.08) & 67/93 (72.04) & $<0.01$ \\
\hline Vancomycin & 397/398 (99.75) & $254 / 254(100.00)$ & 0.42 \\
\hline Levofloxacin & 217/399 (54.39) & $162 / 254(63.78)$ & $<0.01$ \\
\hline
\end{tabular}

Table 5 Trend of antibiotic susceptibility for $S$. aureus between the two phases

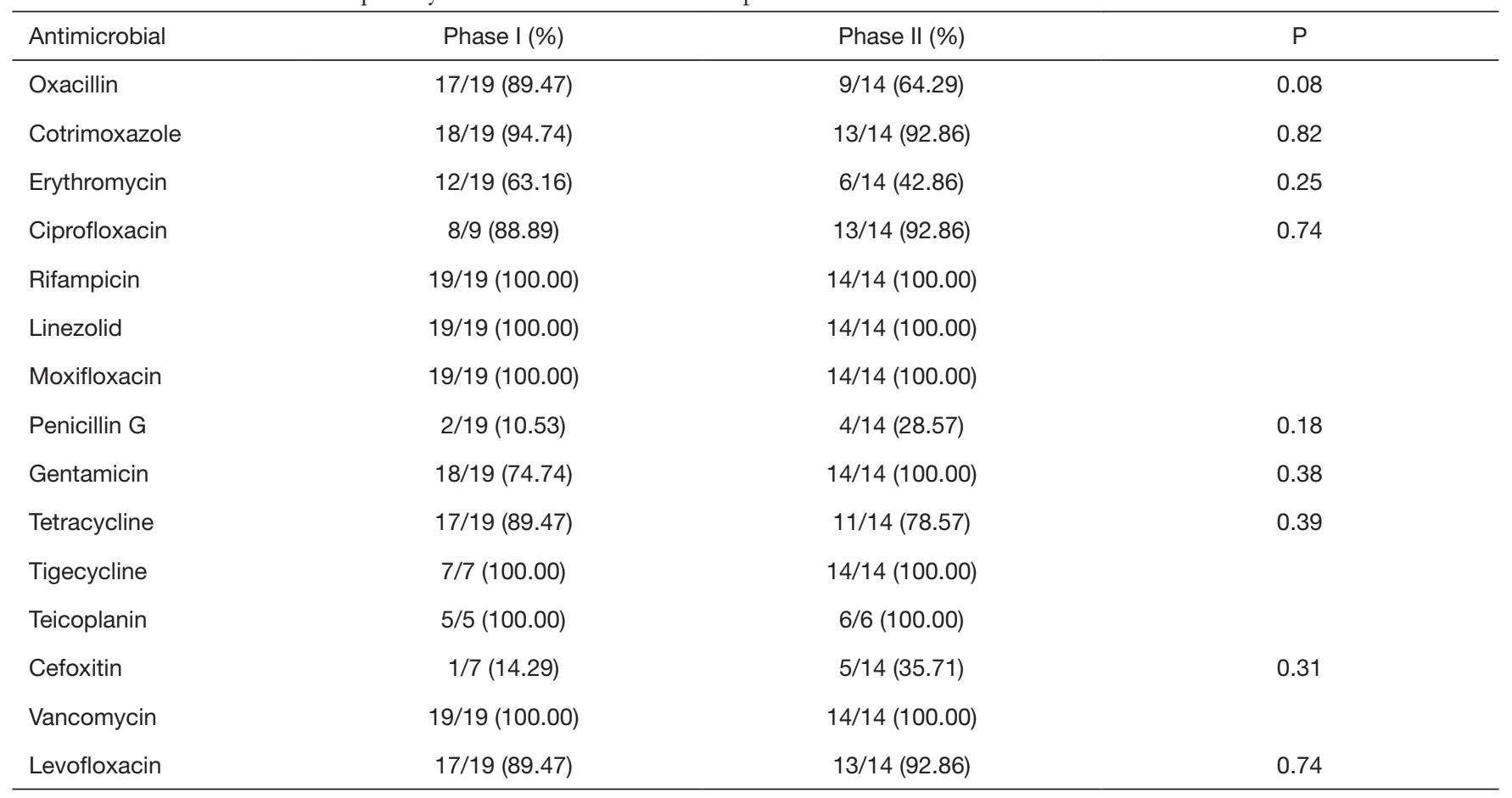


Table 6 Trend of antibiotic susceptibility for K. pneumoniae between the two phases

\begin{tabular}{|c|c|c|c|}
\hline Antimicrobial & Phase I (\%) & Phase II (\%) & $\mathrm{P}$ \\
\hline Ampicillin & $0 / 57(0)$ & $0 / 66(0)$ & \\
\hline Aztreonam & 7/34 (20.59) & 35/66 (53.03) & $<0.01$ \\
\hline Ertapenem & $34 / 34(100.00)$ & 49/49 (100.00) & \\
\hline Ciprofloxacin & 33/34 (97.06) & 62/66 (93.94) & 0.96 \\
\hline Meropenem & 23/23 (100.00) & $13 / 19(68.42)$ & $<0.01$ \\
\hline Piperacillin & $1 / 15(6.67)$ & $3 / 49(6.12)$ & 0.89 \\
\hline Piperacillin/tazobactam & $61 / 61(100.00)$ & 49/66 (74.24) & $<0.01$ \\
\hline Cefuroxime & $5 / 34(14.71)$ & $5 / 36(13.89)$ & 0.92 \\
\hline Cefoperazone/sulbactam & $51 / 69(73.91)$ & 28/65 (43.08) & $<0.01$ \\
\hline Ceftriaxone & $7 / 34$ (20.59) & $13 / 65(20.00)$ & 0.92 \\
\hline Cefotaxime & $1 / 23(4.35)$ & $5 / 17(29.41)$ & $<0.01$ \\
\hline Ceftazidime & $16 / 57(28.07)$ & 28/66 (42.42) & 0.13 \\
\hline Cefotetan & $34 / 34(100.00)$ & $50 / 66(75.76)$ & $<0.01$ \\
\hline Cefoxitin & $53 / 57$ (92.98) & 43/66 (65.15) & $<0.01$ \\
\hline Imipenem & $16 / 16(100.00)$ & $52 / 66(78.79)$ & $<0.01$ \\
\hline
\end{tabular}

mortality due to NS remain a major concern in neonates (7). NS may not have specific signs and symptoms; as such, a delay in diagnosis and treatment contributes to higher morbidity and mortality. Sepsis-related mortality is preventable with time-sensitive empiric antibiotic therapy, which is decided based on the periodic epidemiological survey of local bacterial flora and their antibiotic susceptibility patterns. Causative organisms vary in different regions. Therefore, epidemiologic studies are of great importance in each region. This study retrospectively analyzed the blood culture of positive cases of early-onset sepsis to determine the changing trends of causative bacteria and antibiotic susceptibility in the past decade.

Previous studies emphasized the pivotal role of Gramnegative bacteria, such as $E$. coli and $K$. pneumoniae, as NS-causing pathogens (8-10). However, the main NScausing pathogens, based on reported cases in China, were Gram-positive organisms (11). In the present study,
Gram-positive bacteria were more common than Gramnegative bacteria during phases I and II. However, we observed a significant decrease in the number of Grampositive bacteria during phase II. This decrease was mainly attributed to the decrease in CoNS and the increase in E. coli. Although the proportion of CoNS decreased over time, it remained the major pathogen, consistent with previous study (12). Identifying CoNS is often challenging because most CoNS-positive blood cultures are deemed contaminated $(13,14)$. CoNS cause NS when medical devices penetrate the skin and mucosal barriers. The increasing use of invasive medical devices, including central venous catheters, has increased the prevalence of this strain. The National Institute of Child Health and Human Development Neonatal Research Network published specific criteria to define CoNS sepsis (15). Two positive blood cultures are required to diagnose cultureproven sepsis. In the present study, patients with CoNS 
Table 7 Trend of antibiotic susceptibility for E. coli between the two phases

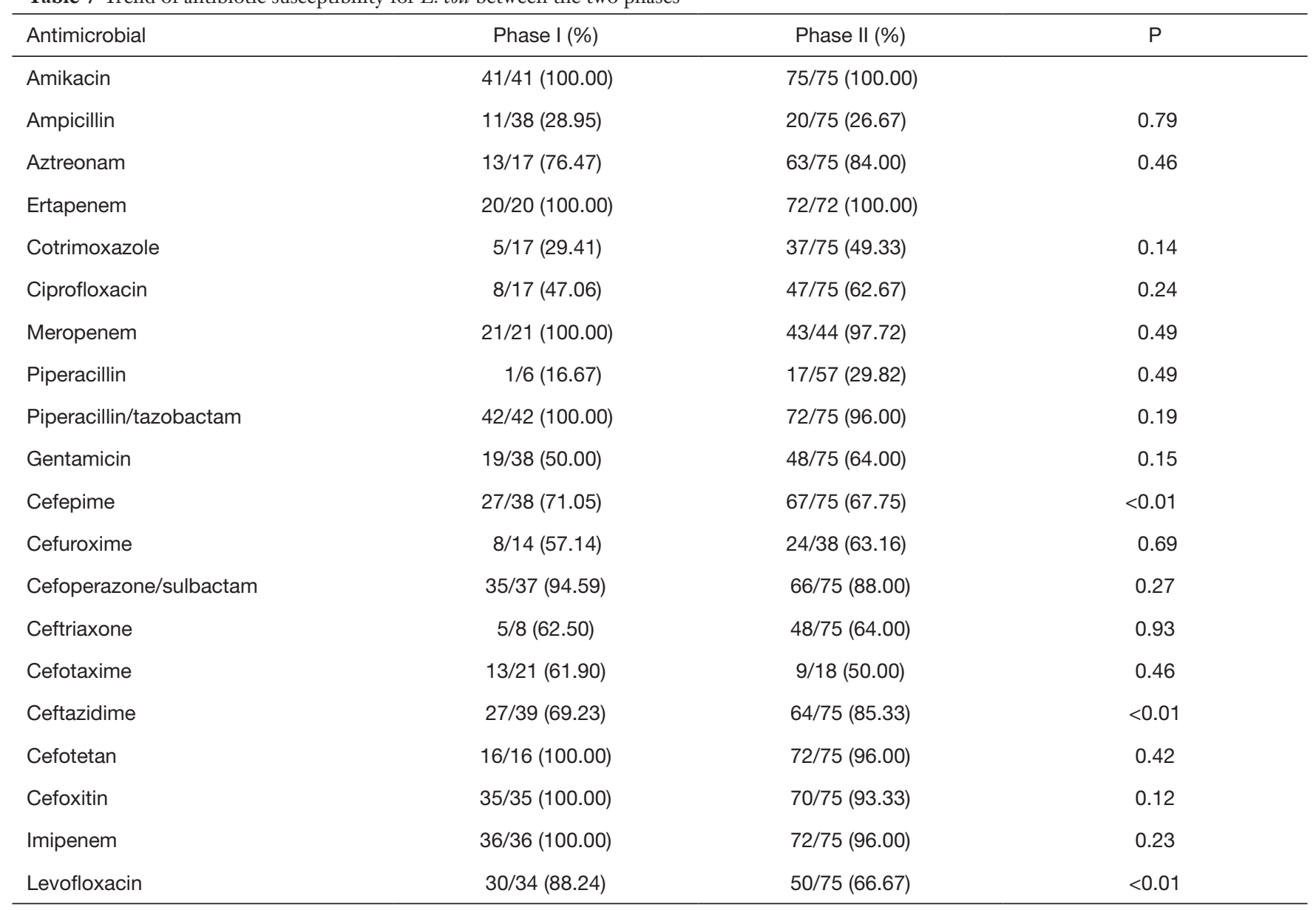

had positive paired blood cultures and were clinically symptomatic for sepsis, confirming the absence of contamination. The decline in the number of CoNS was probably due to improvements in aseptic and disinfection procedures. No significant differences in the number of S. aureus were found between the two phases. Furthermore, the proportion of Streptococcus species increased, which was contradictory to previous reports (16). Our study also demonstrated a significant decrease in the proportion and frequency of Enterococcus species. For Gram-negative organisms, K. pneumoniae was the predominant species initially in phase I but was replaced by $E$. coli in phase II. Meanwhile, the proportion and frequency of Enterobacter and Acinetobacter were significantly higher in phase II than those in phase I. These findings indicate a paradigm shift in the etiology of NS in recent years.

We also detected a significant change in the susceptibility to antibiotics during the last decade. Our results revealed that Gram-positive bacteria exhibited the highest resistance to penicillin, oxacillin, and erythromycin and remained sensitive to vancomycin, tigecycline, and teicoplanin. CoNS exhibited approximately $90 \%$ resistance toward penicillin and oxacillin. Based on the results, the use of ampicillin, penicillin G, and first or second-generation cephalosporins, which were previously recommended as empiric antibiotics for the treatment of NS, is probably unnecessary due to their extremely low sensitivity. The WHO recommends first-line antibiotics, including ampicillin and aminoglycosides, for the treatment of NS $(17,18)$. However, ampicillin showed alarming resistance in the present study. Meanwhile, considering the extensive use of thirdgeneration cephalosporines, we observed high antimicrobial resistance among Gram-positive and Gram-negative bacteria, including rapid resistance in $K$. pneumoniae during phase II. The susceptibility to $\beta$-lactamase inhibitors also significantly decreased in $K$. pneumoniae during phase II. 
A hospital used fluoroquinolones to treat Gram-negative infections until $\beta$-lactam plus $\beta$-lactamase inhibitors were available for management due to the high resistance observed to these antibiotics (19). This study found that Gram-negative bacteria remained highly sensitive to quinolones throughout the study period. Therefore, the therapeutic role of fluoroquinolones may gain considerable importance in the future.

Zaidi et al. (17) reviewed 11,471 blood cultures from developing countries in South-East Asia and recommended imipenem and amikacin for the initial treatment of suspected sepsis in hospitalized neonates. Amikacin is effective against Gram-negative bacteria but is rarely used for NS because it can cause kidney and ear-associated toxicity (11). In the present study, the carbapenemresistance significantly increased in $K$. pneumoniae during phase II. The reduced susceptibility to meropenem and imipenem was attributed to their over-prescription in this population, emphasizing the importance of thirdgeneration cephalosporin therapy for these patients. The proportion of $E$. coli significantly increased during phase II and showed a notable resistance pattern. E. coli was resistant to ampicillin and sensitive to cefoperazone/sulbactam, piperacillin/tazobactam, cefotetan, and cefoxitin, all of which are commonly administered antibiotics. According to our findings, the resistance of E. coli against cephalosporins remained relatively unchanged over the last decade. This change in the sensitivity pattern of antimicrobials could be attributed to the fact that microorganisms tend to become resistant to commonly used antibiotics while remain sensitive to the rarely used ones.

\section{Conclusions}

In recent years, microbial profiles in NS have significantly changed, including a reduction in CoNS and an increase in E. coli. An increasing trend of antibiotic resistance to commonly used and available drugs has been observed. The resistance toward carbapenems has considerably increased. Antibiotic susceptibility patterns should be assessed in different phases. Each hospital should implement a unique antibiogram program that assesses options for empirical antibiotic therapy. This study had certain limitations, most notably the lack of correlation of our findings to clinical features and the limited representativeness of the sample. However, efforts to change the bacteriological profiles and antibiotic susceptibility patterns over 10 years and the large sample size were the strengths of the study.

\section{Acknowledgments}

We thank the contributions of nurses at the Children's Hospital of Soochow University, in providing pathogens and, the collection of specimens. We also thank the support of the colleagues of the Department of Microbiology in our hospital.

Funding: This work was supported by the Jiangsu Province Key Research and Development of Special Funds in China (No. BE2015644); the Jiangsu Province Women and Children Health Research Project (No. F201750); and the Department of Pediatrics Clinical Center of Suzhou City of China (No. Szzx201504).

\section{Footnote}

Reporting Checklist: The authors have completed the STROBE reporting checklist. Available at http://dx. doi. org/10.21037/tp-20-115

Data Sharing Statement: Available at http://dx. doi. org/10. 21037/tp-20-115

Conflicts of Interest: All authors have completed the ICMJE uniform disclosure form (available at http://dx. doi. org/10. 21037/tp-20-115). The authors have no conflicts of interest to declare.

Ethical Statement: The authors are accountable for all aspects of the work in ensuring that questions related to the accuracy or integrity of any part of the work are appropriately investigated and resolved. The study was conducted in accordance with the Declaration of Helsinki (as revised in 2013). The study was approved by the Clinical Trial Ethics Review Committee of Children's Hospital of Soochow University (No. 2019LW019) and individual consent for this retrospective analysis was waived.

Open Access Statement: This is an Open Access article distributed in accordance with the Creative Commons Attribution-NonCommercial-NoDerivs 4.0 International License (CC BY-NC-ND 4.0), which permits the noncommercial replication and distribution of the article with the strict proviso that no changes or edits are made and the original work is properly cited (including links to both the formal publication through the relevant DOI and the license). See: https://creativecommons.org/licenses/by-nc$\mathrm{nd} / 4.0 \%$. 


\section{References}

1. Chiesa C, Panero A, Osborn JF, et al. Diagnosis of neonatal sepsis: a clinical and laboratory challenge. Clin Chem 2004;50:279-87.

2. Sundaram V, Kumar P, Dutta S, et al. Blood culture confirmed bacterial sepsis in neonates in a North Indian tertiary care center: changes over the last decade. Jpn J Infect Dis 2009;62:46-50.

3. Roy MP, Bhatt M, Maurya V, et al. Changing trend in bacterial etiology and antibiotic resistance in sepsis of intramural neonates at a tertiary care hospital. J Postgrad Med 2017;63:162-8.

4. Afsharpaiman S, Torkaman M, Saburi A, et al. Trends in incidence of neonatal sepsis and antibiotic susceptibility of causative agents in two neonatal intensive care units in tehran, I.R iran. J Clin Neonatol 2012;1:124-30.

5. Marzban A, Samaee H, Mosavinasab N. Changing trend of empirical antibiotic regimen: experience of two studies at different periods in a neonatal intensive care unit in Tehran, Iran. Acta Med Iran 2010;48:312-5.

6. Pokhrel B, Koirala T, Shah G, et al. Bacteriological profile and antibiotic susceptibility of neonatal sepsis in neonatal intensive care unit of a tertiary hospital in Nepal. BMC Pediatr 2018;18:208.

7. Abdellatif M, Al-Khabori M, Rahman AU, et al. Outcome of Late-onset Neonatal Sepsis at a Tertiary Hospital in Oman. Oman Med J 2019;34:302-7.

8. Ghotaslou R, Ghorashi Z, Nahaei MR. Klebsiella pneumoniae in neonatal sepsis: a 3-year-study in the pediatric hospital of Tabriz, Iran. Jpn J Infect Dis 2007;60:126-8.

9. Macharashvili N, Kourbatova E, Butsashvili M, et al. Etiology of neonatal blood stream infections in Tbilisi, Republic of Georgia. Int J Infect Dis 2009;13:499-505.

Cite this article as: Tang XJ, Sun B, Ding X, Li H, Feng X. Changing trends in the bacteriological profiles and antibiotic susceptibility in neonatal sepsis at a tertiary children's hospital of China. Transl Pediatr 2020;9(6):734-742. doi: 10.21037/tp20-115
10. Ullah O, Khan A, Ambreen A, et al. Antibiotic Sensitivity pattern of Bacterial Isolates of Neonatal Septicemia in Peshawar, Pakistan. Arch Iran Med 2016;19:866-9.

11. Dong H, Cao H, Zheng H. Pathogenic bacteria distributions and drug resistance analysis in 96 cases of neonatal sepsis. BMC Pediatr 2017;17:44.

12. Guo J, Luo Y, Wu Y, et al. Clinical Characteristic and Pathogen Spectrum of Neonatal Sepsis in Guangzhou City from June 2011 to June 2017. Med Sci Monit 2019;25:2296-304.

13. Bizzarro MJ, Shabanova V, Baltimore RS, et al. Neonatal sepsis 2004-2013: the rise and fall of coagulase-negative staphylococci. J Pediatr 2015;166:1193-9.

14. Cui J, Liang Z, Mo Z, et al. The species distribution, antimicrobial resistance and risk factors for poor outcome of coagulase-negative staphylococci bacteraemia in China. Antimicrob Resist Infect Control 2019;8:65.

15. Stoll BJ, Hansen N, Fanaroff AA, et al. Late-onset sepsis in very low birth weight neonates: the experience of the NICHD Neonatal Research Network. Pediatrics 2002;110:285-91.

16. Acquah SE, Quaye L, Sagoe K, et al. Susceptibility of bacterial etiological agents to commonly-used antimicrobial agents in children with sepsis at the Tamale Teaching Hospital. BMC Infect Dis 2013;13:89.

17. Zaidi AK, Huskins WC, Thaver D, et al. Hospitalacquired neonatal infections in developing countries. Lancet 2005;365:1175-88.

18. Fuchs A, Bielicki J, Mathur S, et al. Reviewing the WHO guidelines for antibiotic use for sepsis in neonates and children. Paediatr Int Child Health 2018;38:S3-S15.

19. Bhattacharjee A, Sen MR, Prakash P, et al. Increased prevalence of extended spectrum beta lactamase producers in neonatal septicaemic cases at a tertiary referral hospital. Indian J Med Microbiol 2008;26:356-60. 